\title{
La interculturalidad como nuevo objeto: Esbozo de un planteamiento transmoderno
}

\author{
Interculturality as a new object: Outline of a Transmodern Approac
}

David Añazco ${ }^{1}$

\section{Resumen}

La interculturalidad entendida como la síntesis de contradicciones entre múltiples expresiones e identidades culturales no puede abstraerse del viejo dilema filosófico, y además político, que implica la discusión de la modernidad versus postmodernidad. En esta propuesta argumento que lo intercultural se manifiesta de manera problemática en ambas matrices de pensamiento; en la modernidad por su sentido común totalizador, y la postmodernidad al encubrir un marco común de referencia. Frente a ello, esbozo un planteamiento vinculado a la tesis de la transmodernidad. Donde sería posible diálogo entre iguales, no como una caridad, sino como una exigencia de los sectores históricamente marginados. Para la construcción de esta posición, movilizo el esfuerzo teórico que se realiza desde la Teoría Histórico Cultural de la Actividad (CHAT) para insertar lo psicológico en un campo de mayor complejidad: la actividad intersubjetiva. Trabajo con la hipótesis del aprendizaje expansivo de la CHAT para caracterizar lo intercultural como un nuevo objeto de aprendizaje colectivo producto de la colisión entre posiciones. Por lo tanto, la interculturalidad es una idea movilizadora que se elabora de manera progresiva como resultado de la gestión del aprendizaje y diálogo cultural.

Palabras Clave: Interculturalidad; Teoría Histórico Cultural de la Actividad (CHAT); aprendizaje expansivo; transmodernidad; postmodernidad; modernidad.

\section{Abstract}

Interculturality is understood as the synthesis of contradictions between multiple expressions and cultural identities that cannot be separated from the old philosophical, and also political dilemma, which implies the discussion of modernity versus postmodernity. In this proposal, I argue that interculturality manifests itself in a problematic way in both matrixes of thought; in modernity for its totalizing common sense, and postmodernism by covering up a common frame of reference. Faced with this, I outline an approach linked to the thesis of transmodernity, where dialogue between equals would be possible, not as a charity, but as a demand of historically marginalized sectors. For the construction of this position, I mobilize the theoretical

1 Master en Psicología de la Educación. Docente - Investigador de la Universidad Nacional de Educación (UNAE- Ecuador). david. anazco@unae.edu.ec. https://orcid.org/oooo-0o03-0619-2835

Recibido: 11/03/2019 Aprobado: 27/06/2019

Añazco, D. (2019). La interculturalidad como nuevo objeto: Esbozo de un planteamiento transmoderno. Ciencia E Interculturalidad, 24(01), 106-119. https://doi.org/10.5377/rci.v24i01.8006 
effort that is made from the Historical Cultural Theory of Activity (CHAT) to insert the psychological in a field of greater complexity: the intersubjective activity. I work with the hypothesis of expansive learning of the CHAT to characterize the intercultural as a new object of collective learning product of the collision between positions. Therefore, interculturality is a mobilizing idea that is developed progressively as a result of the learning and cultural dialogue management.

Keywords: Interculturality; Historical Cultural Theory of Activity (CHAT); expansive learning; transmodernity; postmodernity; modernity.

\section{Introducción}

En "El abrazo de la serpiente" (Guerra, 2015) se narra la historia de Karamakate, único chamán amazónico sobreviviente de la etnia ocaina. La película se base en el diario del etnobotánico alemán Theodor Koch-Grünberg, y su homólogo estadounidense Richard Evan Schultes. En el desarrollo, éste último se inserta en la Amazonía colombiana y se encuentra con Karamakate, quienes, en la búsqueda de la planta yakruna, acuden a una extraña comunidad con rasgos católicos e indígenas donde el único no indígena habla portugués y es tratado como el hijo de Dios. Escenas caóticas: «Esto es una locura» dice el personaje Richard Evan, «ahora estás en el peor de ambos mundos» responde el viejo Karamakate.

¿Hacia dónde nos dirigimos cuándo planteamos la interculturalidad como objetivo de la educación? Esa es la pregunta catalizadora del presente texto, acompañada de, qué garantiza que el planteamiento que hagamos no nos sumerja en el "peor de los mundos", sino en uno, un poco mejor que el presente. Es casi una verdad de Perogrullo señalar que la educación podría ser un motor de cambio y que es necesario apostar a sus potencialidades. Sin embargo, la reconfiguración de la sociedad nos plantea evidenciar algunos desplazamientos y crisis. Por ejemplo, a nivel teórico necesitamos considerar un sujeto diferente (sujeto postmoderno²); y además, una nueva educación que - hoy más que nunca sabemos - no puede reducirse a la escuela, ni a la formalidad institucional. En consecuencia, crisis de nuestras maneras de entender la subjetividad y sus cambios; y crisis del sentido de la educación formal, en tanto sigue respondiendo a un modelo de sociedad industrializada (Collins \& Halverson, 2010).

Este trabajo es el resultado de mi experiencia como estudiante de postgrado en psicología de la educación en la Universidad Autónoma de Barcelona, en especial en clases de psicología cultural con el Grupo de investigación sobre desarrollo humano, intervención social e interculturalidad que buena parte de su experiencia se fundamenta en el trabajo con comunidades gitanas en Cataluña. El debate sobre la interculturalidad

2 El uso del término se pone de manifiesto en contraposición al sujeto moderno de identidad fija y cultura esencial. En ese sentido, refiere a un estado teórico que considera la(s) identidade(s) como un producto de la participación en sistemas de actividad específicos, lo cual la hace maleable y posibilita su transformación de manera continuada. 
en Europa me cautivó en especial porque llegué a éste en un momento de discusión sobre las migraciones y crisis de refugiados. Y, también, porque provenía de un país como Ecuador donde el último proceso constituyente (2008) había reconocido un estado plurinacional e intercultural, al menos de modo formal. Por ello presté mayor atención en cómo desde la psicología podría reflexionar sobre lo intercultural. Para ese momento aún mis lecturas sobre la decolonialidad y epistemologías del sur aún eran muy pobres, condición que se ha ido resolviendo como mi retorno a Ecuador para trabajar en la Universidad Nacional de Educación (UNAE), donde emerge un importante debate sobre la interculturalidad, el indigenismo y la Educación Intercultural Bilingüe (EIB). Estas experiencias del último año (2018) nutrieron mi visión sobre las relaciones intersubjetivas - que ahora puedo asumir como relaciones culturales. Comentar esto no tiene ningún afán biográfico, busca situar el contexto de la interculturalidad a través del sentido que tiene para mí. Además, al trazar esta trayectoria intento transparentar mi posicionamiento.

Así pues, en este texto defiendo la tesis de que la interculturalidad es una resultante favorecida a partir de las colisiones que se pueden generar entre las diversas formas, expresiones, matrices e identidades culturales. Las contradicciones que gestan los procesos de síntesis no pueden asumirse equivalentes o en condiciones de igualdad, debido a que los marcos de referencia en los que se producen las relaciones culturales, de colaboración y conflicto, dan ventaja a las expresiones culturales dominantes, que al final pueden absorber, negar, reprimir y/o aceptar la diferencia como algo decorativo al sentido común de ciudadanía en el Estado Nación. Por lo tanto, el esbozo transmoderno podría interpretarse como una propuesta para una elaboración histórica entre los sectores subalternos y periféricos para confrontar el sentido común dominante, y la estructura que lo sostiene. Lo intercultural no es una apuesta caritativa de parte de la hegemonía, sino una exigencia de parte de los sectores históricamente marginados. Esta elaboración histórica permite cuestionar el sistema y el marco de relaciones, de tal manera que los pondría en crisis. Las fases críticas son las que obligan a reformular y reorganizar las relaciones, prácticas y objetos de actividad cultural. Es decir, generan oportunidades de ciclos expansivos que facultan aprendizajes colectivos, que, en su elaboración progresiva, construyen interculturalidad.

En mi desarrollo ocuparé tres apartados. El primero está destinado a elaborar una representación conceptual sobre el aprendizaje expansivo (Engeström, 2001) y caracterizar la interculturalidad como un nuevo objeto de aprendizaje colectivo. En el segundo apartado presento a la modernidad capitalista y a la postmodernidad como teóricamente problemáticos para pensar en términos de interculturalidad. Finalmente, me posiciono sobre el transmodernismo como espacio teórico donde es posible argumentar y proponer lo intercultural como un nuevo objeto. 


\section{Del subjetivismo a la intersujetividad: la interculturalidad como nuevo objeto desde la visión histórico cultural}

Es reconocido que uno de los principales aportes de la teoría vygoskyana supone para la psicología, salir del foco del individuo e insertarlo en un plano histórico y cultural. Esto ha generado la posibilidad de un encuentro entre la psicología y la sociología (Wertsch, 1988). Más tarde - tras el truncado esfuerzo de la revolución cognitiva por sustituir al conductismo (Bruner, 2006) - el aparecimiento de la psicología cultural abrió la posibilidad de hilvanar relaciones disciplinares que nos permiten generar herramientas de análisis, diálogo y transformación cultural. La tradición histórico cultural asume la cultura como un cuerpo social heredado de la experiencia humana que incluye patrones de comportamiento y artefactos culturales, que sirven para garantizar el desarrollo y acumular experiencia social (Lecusay, Rossen, \& Cole, 2008) A., Rosenbloom, P. S., \& Laird J. E. (1990).

En el marco de la Cultural Historical Activity Theory (CHAT) (Engeström, 2001) una de las herramientas teóricas más potentes que se han elaborado ha sido el aprendizaje expansivo. Para explicarlo debemos recurrir a una explicación de lo que comporta un sistema de actividad (SA). Para ello organizaré la explicación en dos niveles. El primero referente a los elementos del Sistema de Actividad: (1) Artefactos mediadores, materiales y/o simbólicos: productos de la experiencia humana disponibles en la cultura que permiten la modificación de la realidad (en su doble forma ${ }^{3}$ ); y la regulación de la actividad psicológica. (2) Sujetos: aquellos que despliegan intencionadamente la actividad en función de necesidades materiales o psicológicas. (3) Objeto: realidad material/simbólica3. (4) Comunidad: un grupo de sujetos a quienes les entrelazan actividades donde comparten un repertorio de reglas; herramientas mediadoras, sistema de valores, regulaciones. (5) Reglas: valores, tradiciones y/o normas que median la organización de esa comunidad. (6) División del trabajo: roles y tareas que desarrolla cada individuo en un sistema de actividad para la elaboración de un objeto compartido. Finalmente, es necesario destacar que cada elemento constitutivo de un sistema de actividad, al ser un producto cultural, se convierte en un mediador en la elaboración y/o acceso al objeto. En esa línea, es necesario evidenciar la multiplicidad de mediaciones en un SA. Gráfico 1.

3 Sobre este punto es necesario resaltar la importancia de la unidad de análisis que ha señalado Vygtosky: el significado. Esto nos permite sostener la idea de la doble realidad del mundo, material e ideal, unida en el significado (Vygotsky, 1995). 


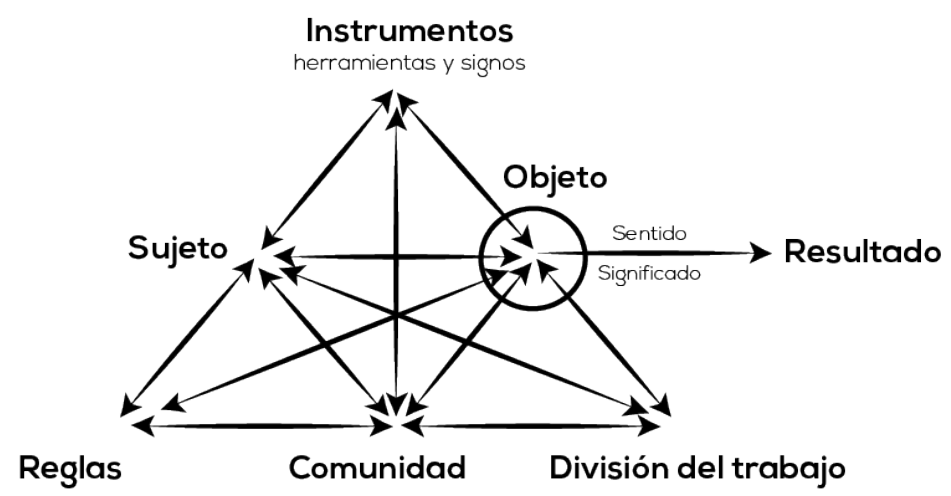

Gráfico No. 1. Representación de la actividad y mediaciones (Engeström \& Sannino, 2010).

El segundo nivel de explicación que propongo refiere a los principios del Sistema de Actividad: 1) el SA como unidad de análisis. 2) Historicidad: Esta refiere a que el SA tiene una permanencia en el tiempo y un devenir histórico en cualquiera de sus elementos, ya sea en las experiencias personales de los sujetos, o bien de las herramientas de mediación, o en la propia comunidad. 3) Multivocidad (multivoiceness): consiste en la presencia simultánea de múltiples puntos de vista, tradiciones e intereses ("voces") en un mismo sistema de actividad, que, a su vez, se ven determinados/ condicionados por normas, historia y reglas propias del SA. El principio de multivocidad es novedoso debido a que esta diversidad de voces no se presentan únicamente intra-sistema, sino también, en relación con otros sistemas de actividad; pero además, dentro de un mismo sujeto (Engeström, 1993). Esto no necesariamente supone un problema, sino que se manifiesta como la expresión de la división del trabajo. 4) Contradicción: Entre los diferentes elementos y las voces del sistema de actividad. El SA entra en crisis, reclamando una reformulación de un objeto, en consecuencia, una modificación de toda la actividad y su orientación. Por lo tanto, es un motor de cambio, evolución o creación de nuevos aprendizajes en los sistemas de actividad. Las contradicciones no deben confundirse con los problemas ni los conflictos, debido a que son tensiones históricas acumuladas, en y entre los sistemas de actividad, que permiten reconceptualizar los objetos. En otras palabras, las contradicciones exigen una re-objetivización de la necesidad. 5) Expansión: Consiste en la reorganización y agudizamiento de la colaboración, debido a las contradicciones que se presentan dentro o con otros sistemas de actividad - ya sea por no compartir necesidades (objetos), o por el cuestionamiento de los valores y normas de la comunidad. Esto garantiza los ciclos de transformación cualitativa, y por consiguiente, la elaboración de nuevos objetos que satisfacen necesidades hacia las que se re-orienta la actividad. Gráfico 2. 


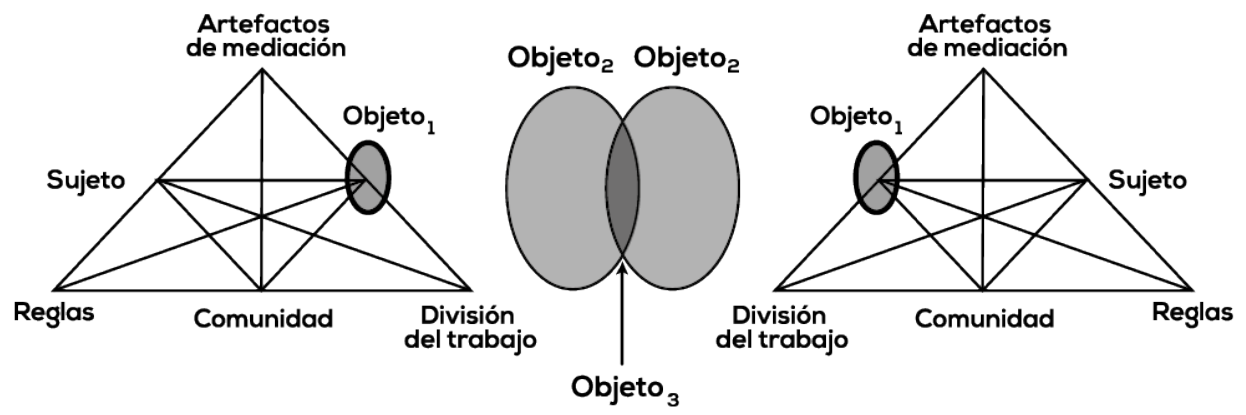

Gráfico No. 2. Dos sistemas de actividad y un nuevo objeto (Engeström, 2001).

Entonces, la tesis del aprendizaje expansivo radica en que todo el sistema de actividad (SA) puede aprender, es decir, no solo aprenden los sujetos, sino los colectivos gracias a la actividad intersubjetiva que despliegan. Esto es posible debido a las contradicciones internas y externas (con otros SA) que sumerge al Sistema de Actividad en periodos de crisis; esto obliga a la reorganización (ciclos expansivos) de la actividad y su reorientación. A medida que estas contradicciones surgen el sistema debe reformular nuevos objetos, que, en su elaboración colectiva, generan aprendizajes. Sobre este punto es necesario resaltar que la representación de la actividad, en la CHAT, constituye una forma de entender la teoría de la actividad ${ }^{4}$ en la obra de Leontyev (1977). Gráfico 1. En este marco, la actividad es entendida como una interacción intencional entre los sujetos y los objetos, donde se produce la transformación de ambos. Y está mediada por herramientas culturales y se orienta a alcanzar (elaborar, construir, transformar) objetos - materiales o simbólicos - que satisfagan necesidades individuales o colectivas.

Para insertar lo intercultural en este marco teórico, preciso de un contraste conceptual entre multiculturalidad e interculturalidad. La primera representa una confluencia de expresiones culturales sin necesariamente entrar en diálogo, reconocimiento y desarrollo de proyectos sociales en conjunto. Mientras que la interculturalidad es definida como "la presencia e interacción equitativa de diversas culturas y la posibilidad de generar expresiones culturales compartidas, adquiridas por medio del diálogo y de una actitud de respeto mutuo" (UNESCO, 2005, p. 5). De esta manera, la interculturalidad no es entendida como un espacio donde coexisten diversas expresiones culturales (manifiestas en los sistemas de actividad), sino como la creación de nuevos espacios de generación cultural para proyectos conjuntos, nuevas expresiones e identidades culturales. En el marco teórico que hemos presentado, la interculturalidad supondría un nuevo producto resultado del encuentro, contradicciones y diálogo

4 Según Engeström (2001) existen tres generaciones de la CHAT: 1) Los principios vygtoskyanos; 2) Teoría de la actividad de Leontiev; y 3) La elaboración de Engeström. 
cultural. En consecuencia, el proceso intercultural es un ciclo expansivo, que permite condiciones para reconfigurar estructuras, nuevos marcos de referencia, es decir, la generación de un nuevo objeto: aprendizaje expansivo.

\section{La modernidad y la postmodernidad como problemas en el planteamiento intercultural. Nudos críticos}

La complejidad y las formas de enfrentar los conceptos que encierra este apartado podrían suscitar largas y variadas discusiones. Sin embargo, tenemos espacios en los que podemos maniobrar sobre el hilo conductor de nuestra argumentación. Un primer nudo crítico es el referido a la noción de alteridad, que se refiere a la existencia y reconocimiento del otro/a. En la modernidad esto supone un problema, porque dado el reconocimiento del otro, este es alguien que debe ser adaptado, formado o civilizado para que pueda participar del proyecto que la totalidad formula. Intrínseco a ello, asociamos la ontología realista: hay una verdad y es cognoscible (Willig, 2013). Por lo tanto, observamos la dirección científica dominante, con pretensión universal, como destino teleológico. Para decirlo de otra manera, el/la otra es alguien que debe ser encarrilado en el destino normalizado hacia la verdad.

El conocimiento es determinante en el marco de la racionalidad científica: quien no está ilustrado, no es moderno ("irracional" "no civilizado"). Este es uno de los enunciados por los que recurre una de las críticas más fuertes desde el postmodernismo, en señalar lo fijo y lo permanente como un problema (Vattimo, 1992) debido a que no permite asumir lo otro como diferente o incluso mejor, sino como insuficiente y subdesarrollado. En los marcos culturales caracterizados por la diversidad esto supone un problema, porque en la modernidad la cultura es asumida como esencial, y la dirección del proyecto de desarrollo estaría preestablecido; y las otras expresiones culturales se ven disueltas. Por lo tanto, lo intercultural no tiene sentido dentro de la modernidad. En esa línea podríamos referirnos a la estrategia de segregación cultural (Lalueza, 2012) como coincidentes con la modernidad, en tanto persecución de la diferencia. Ahora bien, es necesario puntualizar que la modernidad puede tener diversas lecturas. La posición polémica que podemos asumir aquí es subrayar la modernidad con apellido, es decir, modernidad capitalista. Echeverría (1995) plantea: "Entre modernidad y capitalismo existen las relaciones que son propias entre una totalización completa e independiente y una parte de ella, dependiente suya, pero en condiciones de imponerle su manera particular de totalización" (tesis 1).

En ese sentido, podemos subrayar la crítica de la elaboración postmoderna a lo dogmático, a la totalización en sintonía de lo moderno. En contraposición se erige la reivindicación de lo múltiple, lo plural, lo diferente. Esto implica la ruptura de la binariedad, bueno - malo, mejor - peor, desarrollado - subdesarrollado. Desde esta consideración, en el postmodernismo habría la posibilidad de poner en contacto a las diferencias. Empero, el problema es que no las logra poner en diálogo, no se logra 
una tensión equivalente para el desarrollo de proyectos conjuntos y complejos. En consecuencia, podríamos indicar un correlato de la postmodernidad con lo multicultural. Es necesario llamar la atención sobre este punto: la estrategia multiculturalista implica el respeto a la diferencia dentro de un mismo espacio de ciudadanía (Lalueza, 2012) sin necesariamente trascenderlo.

La cuestión que desprendemos de lo que antecede sería ¿por qué no es posible un planteamiento intercultural desde la postmodernidad? Mi argumento es que las diferencias en realidad se relacionan en condiciones de desigualdad, y esto no garantiza la alteridad y el diálogo pleno o, como he manifestado, una tensión equivalente que permita una contradicción favorable. Al respecto me parece fundamental la aportación de Ogbu (1993) en relación al estatus cultural en función del marco de referencia. Y, además, reconsiderar los conceptos: (1) Minoría cultural involuntaria: aquellas que se incorporan por razones en contra de su voluntad a un nuevo marco cultural; y (2) Minoría cultural voluntaria: aquellas que eligen incorporarse en un nuevo marco. Debido a esto podemos indicar asimetrías en las relaciones culturales en consideración de los marcos de referencia en los que se relacionan. En ese caso nos referiríamos a interculturalidad en términos de integración/acceso al marco de referencia. En otras palabras, supondría la transformación de los diferentes patrones de comportamiento, identidades, herramientas culturales, normas de una comunidad en función de un marco de referencia ${ }^{5}$. Esto a mi juicio acoge el planteamiento del melting pot que en Estados Unidos presumía, supuestamente de interculturalidad, pero en la práctica implicó la asimilación de la cultura anglosajona por parte de las expresiones culturales minoritarias e históricamente segregadas (Lalueza, 2012).

En el intento de caracterizar tanto a la modernidad y postmodernidad como teóricamente problemáticos respecto de la interculturalidad, señalamos un segundo nudo crítico: los grandes discursos. La crítica postmoderna sugiere que se han agotado las intenciones de la modernidad por transformar radicalmente (desde la raíz) las formas de la vida social (arte, pensamiento, ciencia, política) expresadas en meta relatos. Y en ese sentido, arguye que las grandes narrativas de la modernidad, y el futuro que proyectaban, han sido liquidados ${ }^{6}$ (Lyotard, 2004).

Más allá de la caracterización específica de los meta-relatos, lo que me interesa resaltar es en el contenido central que las fundamenta. Y con esa intención, citar lo señalado por Lyotard (1987) quien años más tarde de la primera caracterización de los meta-relatos, y de una forma autocrítica, señala: "Los meta - relatos...no buscan

5 Propongo plantear a la globalización como marco de referencia que, a diferencia de otros planteamientos históricos, no es fruto de un esfuerzo teórico, sino de una revolución tecnológica; la mundialización del capital y la producción. Y en ese sentido se restituye lo binario: globalizado/universal - excluido/marginal.

6 La tesis que desarrolla Echeverría (1995) gira en torno a la crítica de la modernidad capitalista, en donde el discurso del "socialismo real", no la anuló, simplemente la hizo más débil. Por lo tanto, se abre la posibilidad de postular una modernidad post-capitalista (tesis 2) lo cual implica crear, en un marco crítico, un concepto de revolución que supere al idealista - romántico (Echeverría, 2011) 
la referida legitimidad en un acto originario fundacional, sino en un futuro que se ha de producir, es decir, en una Idea a realizar" pp. 30. (el subrayado es mío). Este bloqueo a una posible condición futura, también es considerado por Castro-Gómez (2011) quien en referencia a una caracterización de la postmodernidad en la situación latinoamericana refiere: "el presente es el único horizonte de significación, a falta de un proyecto futuro" (pp. 27).

Por una parte, hemos intentado exponer que la interculturalidad no puede operar en la modernidad capitalista considerando el carácter dogmático respecto de una cultura fija/universal donde las otras culturas son objetos de civilización y/o marginalización. Por otra parte, hemos intentado señalar que la interculturalidad no transita en la postmodernidad, debido a la desigualdad y asimetría de relaciones entre los diferentes. Es lo que hemos llamado el primer nudo crítico. Pero, además, desde nuestro marco teórico, cuando argumentamos que la interculturalidad es un nuevo objeto producto de la colisión/contradicción/reorganización dentro o con otros sistemas de actividad, si esa contradicción no es identificada, y el nuevo objeto discutido y organizado, la contradicción no va a ser superada. En otras palabras, la interculturalidad no es una resultante fortuita de la contradicción entre sistemas de actividad. Por tanto, debe ser direccionada. Y en esa lógica, supone intención sentido/ ideología/futuro, elementos que la postmodernidad intenta aplacar. Por lo tanto, la intención intercultural, como objetivo fijado por los diferentes sectores sociales, puede considerarse en el marco de lo expuesto por Lyotard (1987) como un meta - relato ("futuro que se ha de producir, es decir, en una Idea a realizar"). Eso es lo que hemos llamado como segundo nudo crítico.

En síntesis, en lo que respecta la interculturalidad como nuevo objeto, crisis de la modernidad, pero también de la postmodernidad.

\section{A modo de conclusión: esbozo de un planteamiento transmoderno sobre la interculturalidad}

Considero que aquella sentencia de Lyotard (2004) sobre el fracaso de los metarelatos, o lo que podríamos considerar como los grandes proyectos de desarrollo, es una hipótesis con fuerza; sin embargo, en esta transición histórica de la sociedad hacia la agudización de la globalización a través de lo virtual, en donde lo semiótico adquiere una fuerza insoslayable, la modernidad capitalista está muy vigorosa. A mi juicio esta fortaleza se produce debido al dogma de que sin la economía de mercado la sociedad no puede desarrollarse, idea que también nutre al occidentalismo como matriz cultural. De ahí que el planteamiento postmodernista, sea iluso y no se ajuste a las circunstancias injustas de diálogo cultural.

Ejemplos contundentes de lo anterior: las limitaciones de sectores políticos de países de la periferia europea, cuando intentan generar nuevos proyectos políticos/ 
culturales alternativos a la centralidad, y a los dictámenes económicos de las principales potencias. Recelo y desconfianza en el mundo occidental hacia otras expresiones culturales como las que manifiestan valores islámicos. Desprecio o uso decorativo de los saberes y expresiones ancestrales, populares y prácticas culturales de grupos históricamente marginados, periféricos y empobrecidos. Negación, subordinación, burlas o cooptaciones académicas a las lenguas indígenas o sus conceptualizaciones. Reverberación del fascismo, en concentraciones y marchas, en oposición a los refugiados sirios y a los migrantes; crisis de la democracia representativa como único o más elevado modo de gestión política, que se percibe en los triunfos de, por ejemplo, Trump y Bolsonaro. En consecuencia, de la globalización capitalista - como nueva totalidad y eje cultural - no podemos esperar sino persecución (material y simbólica) a la diferencia. Y de la postmodernidad, en tanto condición y no como ideología (CastroGómez, 2011), podemos esperar sino, un falso diálogo cultural mientras la matriz de pensamiento occidental donde opera, siga intacta. Como explica Dussel (2017), lo que a veces se nombra como diálogo, es en realidad una relación de dominación y explotación de una cultura sobre otras.

Ahora bien, ¿cómo o en qué marco podemos argumentar el planteamiento intercultural? Considero que a través de dos vías posibles. La primera, siendo capaces de generar una modernidad alternativa post - capitalista (Echeverría, 1995, 2011), no obstante es una vía muy polémica si rastreamos la posible respuesta desde una planteamiento decolonial'. Y la segunda, al interno de un planteamiento transmoderno. Ambos demandan que la intención de generar la interculturalidad como un nuevo objeto, sea trabajada tanto a nivel macro: políticas y sistemas sociales; en lo micro: la escuela, el barrio, la comunidad, y, en los bordes: las prácticas interculturales que se realizan en diferentes sectores sociales, populares e indígenas que deben ser evidenciado. Caminar hacia la interculturalidad no significa descubrirla, sino significa elaboración. Esto demanda organización, diálogo, pactos sociales y políticos, debido a que la superación de los cambios (expansión) no es una resultante de la yuxtaposición de buenas intenciones o contradicciones, sino son productos de una voluntad tácita, como lo argumenta el modelo teórico que hemos expuesto en el primer apartado. Tal como proponen los principales teóricos de la CHAT: la nueva estructura de actividad no cae del cielo (...) requiere de un análisis reflexivo de la estructura de actividad existente: los participantes deben aprender a conocer y comprender lo que quieren superar (Cole \& Engeström, 2001).

En referencia a lo transmoderno, Rodríguez Magda, (2004) ha señalado, en clave hegeliana, que ésta puede presentarse como la síntesis de la modernidad y la

7 Ver por ejemplo: https://desinformemonos.org/hacia-marx-desconocido-hacia-marx-decolonial/. Ramón Grosfoguel: "En el XXI, aún con todos estos marxistas supuestamente que no son ya ortodoxos, siguen repitiendo lo mismo: Se sigue haciendo una ontología del trabajo, se sigue reduciéndolo todo a eso, se sigue pensando que es desde ahí que vas a explicar lo demás. No se ve la modernidad como un proyecto civilizatorio imperialista-colonial, sino como un proyecto de emancipación y entonces empiezan a hablar de un futuro anticapitalista moderno, ¡imagínate!, ¿cómo tú vas a llegar ahí? Y eso lo vemos también en formulaciones de alguien como Bolívar Echeverría, una formulación totalmente eurocéntrica y problemática de todo esto" 
postmodernidad (tesis y antítesis respectivamente). Sin embargo, esto demanda ponerle un apellido a la modernidad que, de ser capitalista, nos llevaría a cuestionarnos si podemos gestionar la postmodernidad como una antítesis válida. Más allá de este problema, me interesa subrayar que la propuesta que intento desarrollar deviene del planteamiento de la transmodernidad de la filosofía de la liberación (Dussel, 2003, 2005, 2017) que supone en principio, un cuestionamiento al occidentalismo como productor de jerarquía en el tratamiento de la diferencia cultural entre occidente y los otros. Además, implica una defensa de la valoración del efecto (Arriscado-Nunes, 2014) de nuestras prácticas, saberes e identidades; desde donde podemos construir un eje de confrontación a los sistemas que ponen en riesgo la vida.

En sentido general, asumir una posición trasnmoderna, demanda, en primera instancia, un contacto mundial en sentido lateral, Sur - Sur que incluya las minorías del centro (por ejemplo, los latinos en Estados Unidos o los africanos en Europa), o bien un contacto nacional-local, de igual forma lateral entre los de abajo o las minorías o grupos subalternos, en lo que podríamos llamar transmodernidad en pequeña escala. Este contacto podría caracterizarse por un diálogo transversal de las diferencias $^{8}$ (Dussel, 2017), en donde se encuentran, y se reconstruyen los movimientos críticos y los sectores marginados de la cultura occidental. Esto, para elaborar nuevas narraciones de nuestros pasados y nuestras proyecciones futuras, a fin de reclamar un diálogo cultural de igual a igual, que esta vez sí, permita generar ciclos expansivos que nos faculten trascender como sociedades hacia la elaboración de un nuevo objeto compartido: la interculturalidad. Esto se conecta al marco teórico histórico cultural, porque el significado de igual a igual, supondría que el nuevo objeto evite una dirección racional (hacia conceptos científicos) sino expansiva, lateral (Cole \& Engeström, 2001; Engeström, 2001).

Pareciera que nos hemos sumergido por un momento en la utopía, empero, no tengo pretensión de negarla. No obstante, no significa otra cosa que cambiar el eje desde donde se desarrollan las propuestas, las intenciones o pretensiones de interculturalidad. ¿Qué supone esto? Que las expresiones culturales en desigualdad de condiciones frente a las culturas dominantes, sean capaces de reorganizar su pasado, es decir, elaborar narrativas cualitativamente potentes y críticas, que les permitan reclamar reconocimiento y un diálogo digno como catalizador del proyecto intercultural (Besley \& Peters, 2011). Al respecto puedo comentar el siguiente aprendizaje: en el año 2018 se desarrolló, en la Universidad Nacional de Educación (UNAE), la primera cátedra indígena. El Taita Luis Enrique Cachicuango comentaba: "un diálogo se construye de «tú a tú», en condiciones semejantes. El diálogo requiere de mucho trabajo a lo interno. Quiero decir que demanda trabajo intracultural”.

8 Según Dusell (2017): "transversal indica aquí ese movimiento que va de la periferia a la periferia; del movimiento feminista a las luchas antirracistas y anticolonialistas. Las Diferencias dialogan desde sus negatividades distintas sin necesidad de atravesar el centro de hegemonía" pp. 284. 
Proponer que la interculturalidad debe construirse desde los sectores excluidos y marginados, porque son estos sectores lo que deben elaborar una posición que reclame el diálogo y no esperarlo, podría juzgarse como una idea que aúna la confrontación y la violencia entre expresiones culturales. Esta sería la expresión temerosa de la hegemonía ante el riesgo de perder su poder. Tal cual ocurre con la paranoia al feminismo, cuando es juzgado de violento por quienes temen perder sus privilegios. La analogía puede extenderse: la igualdad de género, no va a venir de una beta benévola del sistema patriarcal, ni la interculturalidad de cierto rescoldo de bondad del sentido común occidental capitalista.

Así, resulta fundamental la solidaridad con la idea de interculturalidad-bilingüe que sostienen algunas organizaciones andinas en países como Ecuador, que, si bien podríamos expresar que plantean una posición de lo que podríamos definir como visión estrecha de la interculturalidad, ésta se convierte en un espacio potente para la resistencia a la totalización; incluso de lo que es, no es y debería ser la interculturalidad. A mi juicio esta visión estrecha, pero en resistencia, podría orientarse, a través del movimiento transversal señalado, hacia una visión amplia y nueva, es decir, una definición diferente de las relaciones culturales e intersubjetivas. Es decir, la interculturalidad en sentido estrecho puede ser una incubadora de interculturalidad en sentido amplio. De este modo, de la interculturalidad podemos decir simplemente que es síntesis, una novedad (nuevo objeto), y que por ello no puede caracterizarse sino como un concepto vivo, en movimiento.

En un marco cada vez más general, occidental, globalizado y capitalista las relaciones culturales no pueden dejarse a la incertidumbre. En relación al uso de la situación que introduce este texto, cabe señalar que: podría ser que mañana nos despertemos con la certeza de que las relaciones culturales que vivimos sean «juna locura! » y alguien nos secunde: «estamos en el peor de todos los mundos». En contrapartida, podemos explorar nuestras capacidades para plantearnos las posibilidades de un mundo distinto. Me parece que la épica - como deseo que construye sentido - es una condición indispensable para la interculturalidad. En definitiva, hay que construir un horizonte de significación futura, a partir de la reorganización de nuestros pasados (a modo de Walter Benjamín, cepillar la historia a contrapelo), en donde la actuación en el presente nos permita aprender a generar oportunidades para la interculturalidad como condición futura.

\section{Agradecimiento}

Esta publicación obtuvo el financiamiento de: El Fondo de Asistencia Internacional de los Estudiantes y Académicos Noruegos, (SAIH). 


\section{Lista de referencias}

Arriscado-Nunes, J. (2014). El rescate de la epistemología. En Boaventrura de Sousa Santos \& M. Meneses (Eds.), Epistemologías del Sur (perspectivas) (pp. 219-244). Madrid: Akal.

Besley, T., \& Peters, M. A. (2011). Interculturalism, ethnocentrism and dialogue. Policy Futures in Education, 9(1), 1-12. https://doi.org/10.2304/pfie.2011.9.1.1

Bruner, J. (2006). Actos de significado mas alla de la revolucion cognitiva. Madrid: Alianza Editorial.

Castro-Gómez, S. (2011). Crítica a la razón latinoamericana (2da ed.). Bogotá: Editoral Pontificia Universidad Javeriana.

Cole, M., \& Engeström, Y. (2001). Enfoque histórico-cultural de la cognición distribuida. En G. Salomon (Ed.), Cogniciones distribuidas: Consideraciones psicológicas y educativas (pp. 23-74). Buenos Aires: Amorrortu Editores.

Collins, A., \& Halverson, R. (2010). The second educational revolution: Rethinking education in the age of technology. Journal of Computer Assisted Learning, 26(1), 18-27. https://doi.org/10.1111/j.1365-2729.2009.00339.x

Dussel, E. (2003). La filosofía de la liberación ante el debate de la postmodernidad y los estudios latinoamericanos. Dorando Michelini (Comp.) Filosofía de La Liberación. Balance y Perspectiva, 30, 1-2.

Dussel, E. (2005). Transmodernidad e interculturalidad (Interpretación desde la Filosofía de la Liberación). México City: UAM.

Dussel, E. (2017). La nueva edad del mundo. Transmodernidad. En Filosofías del Sur. Descolonización y transmodernidad (pp. 257-294). Buenos Aires: Akal/Inter Pares.

Echeverría, B. (1995). Modernidad y capitalismo (15 tesis). Ilusiones de La Modernidad, 133-197.

Echeverría, B. (2011). Modernidad y revolución. En F. Tinajero (Ed.), Bolívar Echeverría, ensayos políticos (pp. 159-168). Quito: Ministerio de coordinación de la política (Ecuador). Recuperado de: http://www.bolivare.unam.mx/ensayos/Libro Pensamiento Politico 1.pdf

Engeström, Y. (1993). Developmental studies of work as a testbench of activity theory: The case of primary care medical practice. Understanding Practice: Perspectives on Activity and Context, 64-103. 
Engeström, Y. (2001). Expansive Learning at Work: toward an activity theoretical reconceptualization. Journal of Education and Work, 14(1), 133-156. https:// doi.org/10.1080/13639080020028747

Engeström, Y., \& Sannino, A. (2010). Studies of expansive learning: Foundations, findings and future challenges. Educational Research Review, 5(1), 1-24. https:// doi.org/10.1016/j.edurev.2009.12.002

Guerra, C. (2015). El abrazo de la serpiente. Colombia: Oscilloscope Pictures.

Lalueza, J. L. (2012). Modelos psicológicos para la explicación de la diversidad cultural. Cultura y Educacion, 24(2), 149-162. https://doi.org/10.1174/113564012804932119

Lecusay, R., Rossen, L., \& Cole, M. (2008). Cultural-historical activity theory and the zone of proximal development in the study of idioculture design and implementation. Cognitive Systems Research, 9(1-2), 92-103. https://doi.org/10.1016/j. cogsys.2007.06.012

Leontyev, A. N. (1977). Activity and consciousness. Philosophy in the USSR, Problems of Dialectical Materialism.

Lyotard, J.-F. (1987). La Posmodernidad : explicada a los niños. Barcelona : Gedisa.

Lyotard, J.-F. (2004). La Condició postmoderna : informe sobre el saber. Barcelona : Angle.

Ogbu, J. U. (1993). Differences in cultural frame of reference. International Journal of Behavioral Development, 16(3), 483-506.

Rodríguez Magda, R. M. (2004). Transmodernidad. Rubí : Anthropos.

UNESCO. (2005). Convención sobre la protección y promoción de la diversidad de las expresiones culturales. Paris, Francia: UNESCO. Recuperado de: http://unesdoc. unesco.org/images/oo14/o01429/142919s.pdf

Vattimo, G. (1992). Posmoderno: ¿ una sociedad transparente? Debate Feminista, 5, 120-128.

Wertsch, J. V. (1988). Vygotsky y la formación social de la mente. Barcelona: Paidós.

Willig, C. (2013). Introducing qualitative research in psychology. New York : McGraw-Hill Education/Open University Press. 